\title{
Development of Parametric Technology and Its Application in the Development of Heavy Machine Parts Library
}

\author{
Bei chen ${ }^{1, a^{*}}$, Yan cao ${ }^{2, b}$, Jiang $\mathrm{du}^{3, \mathrm{c}}$ and Miaomiao Zhang ${ }^{4, d}$ \\ ${ }^{1}$ Department of Mechanical and Electronic Engineering, Xi'an Technological University, Xi'an, \\ China

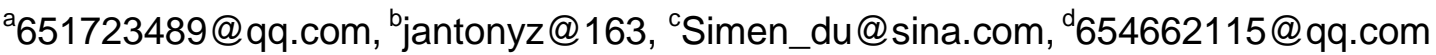

Keywords: Parametric design; Constraint; Research status; CAD

\begin{abstract}
The present situation of the parametric design technique based on constraint, the thought mechanism and advantages and disadvantages of various parametric methods are summarized. Parametric design is to reduce the standard parts of the entire development cycle, to facilitate the management of standard parts data. Through the nut in heavy machine parts as an example, the CATIA V5software is used to carry out parametric design modeling and the standard parts library is established. The results show that the establishment of the standard parts library is practical and effective, and the efficiency is improved. At the same time, the shortcomings of the current research are analyzed, and the future development trend of parametric technology is analyzed and explored.
\end{abstract}

\section{Introduction}

Parametric design is by changing the graphics in one part or several parts of the size, or modifying the component parameters defined, automatically changes to the relevant part of the graph, so as to realize the parametric graphics driver. The parameter driven method is used for the user to modify, delete and design. In the design outline without accurate positioning and setting, the users only need to sketch the outline, and then modify the size value to achieve the final shape, or only the key part of the part is defined as a parameter, realize the product design and optimize the parameters of the amendment. Parametric design has greatly improved the means of modification of graphics, and increased the efficiency of design flexibility and design. At present, parametric design has become one of the most popular application technologies in CAD technology, and it has become an important technical index to evaluate the quality of CAD system.

Parametric design is different from the traditional design in practical engineering design, in order to further application of technology parameters, parametric technology and feature technology, CAD technology integration, combined with other technologies, has important significance for the in-depth study of CAD automation. In order to implement the important technology of standardization, serialization and generalization [1], the CAD system of high efficiency and practical mechanical equipment can be developed with the combination of CAD technology and parametric technology. When the designer inputs the corresponding design parameters, the system can automatically check create parts and assembly, finally can automatically output the parts drawings and assembly drawings and other related design documents, which greatly improves the design efficiency.

CATIA V5 has the functions of parametric design, it can be through the design features and based on sketch to complete parametric modeling of parts, parametric design can also be accomplished through the two development tools. The key lies in the use of parametric modeling parameters, formulas, tables, characteristics of driving pattern has reached the purpose of changing the graphics, the parametric method based on CATIA can be achieved by 3 approaches: (1)the system parameters and dimensions of constraint driven graphics; (2)the user parameters and formula driven graphics;3) using spreadsheet driven graphics[3]. 


\section{Status of Parametric Study}

The nature of the parametric approach is based on the constraints of the product description method. This is because the whole design process of a product is a constraint refinement process, which is a constraint specification, a constraint transformation solution, and a constraint evaluation. Parametric work can be traced back to the early 60s, Sutherland Sketchpad system in his development, the first geometric constraints expressed as nonlinear equations to determine the location of twodimensional geometry. Later in Light, Giscard further developed this idea, and makes it practical. In the application of the numerical iterative method, it was found that many problems such as iterative convergence problems and so on. In this paper, we propose a symbolic method to overcome these problems. However, this method is computationally expensive and has many limitations in practical applications.

Table 1 Comparison of various parametric methods

\begin{tabular}{|c|c|c|}
\hline \multicolumn{2}{|c|}{ Method } & Advantage \\
\hline \multicolumn{2}{|c|}{$\begin{array}{l}\text { Program } \\
\text { parameterization } \\
\text { method }\end{array}$} & $\begin{array}{l}\text { To establish their own parametric } \\
\text { software library or parametric standard } \\
\text { library, the system is user oriented }\end{array}$ \\
\hline $\begin{array}{l}\text { Variable } \\
\text { method } \\
\text { method) }\end{array}$ & $\begin{array}{l}\text { geometry } \\
\text { (algebraic }\end{array}$ & $\begin{array}{l}\text { Can adapt to a wide range of constraint } \\
\text { types, through the simultaneous solution } \\
\text { of the constraint equations can solve the } \\
\text { problem of loop constraints }\end{array}$ \\
\hline $\begin{array}{l}\text { Geometric } \\
\text { reasoning } \\
\text { method } \\
\text { based on }\end{array}$ & $\begin{array}{l}\text { Aldefeld } \\
\text { method }\end{array}$ & $\begin{array}{l}\text { The expression is simple and intuitive, } \\
\text { the separation of knowledge and } \\
\text { processing, the expansion of rule base, } \\
\text { can avoid the instability of numerical } \\
\text { algorithm, and can effectively deal with } \\
\text { the problem of under constraint and over } \\
\text { constraint. }\end{array}$ \\
\hline knowledge & $\begin{array}{l}\text { Sunde } \\
\text { method }\end{array}$ & $\begin{array}{l}\text { Reduce the number of facts and planning } \\
\text { required by the pure expert system, and } \\
\text { can quickly guide the inference to the } \\
\text { right direction. }\end{array}$ \\
\hline
\end{tabular}

Constraint propagation method based on degree of freedom analysis

Method based on A series of parts designed for the same construction process

Based on the auxiliary line method

Graph based parametric method size of structure

Due to the introduction of the concept of degree of freedom, the scale of problem solving is reduced, the search space is effectively controlled, and the reasoning efficiency is improved.

\section{Fast solving speed}

Users do not have to construct the original image in the system-specific module, the algorithm is efficient, easy to implement, the program is not large, portability strong
It is not easy to add new constraint type and entity type, it is difficult to solve complex graphics

Is very sensitive to the constraint equation, the same set of constraints spread in different orders, there may be different solutions or even no solution, or there are multiple solutions, the results are difficult to predict

Lack of flexibility and flexibility; difficult to use the traditional CAD system to generate graphics; can not handle high degree of constraint coupling cycle constraints

It is difficult to define the completeness of the graph constraint set with the help line

Limited use, for complex graphics constraints are limited, can not be fully described 
Therefore, some other methods are proposed, such as artificial intelligence, graph-based parameterization and many more. From the big direction, the parametric design method is divided into two kinds of programming and graphical interaction design methods, graphical interaction is divided into online interactive and offline interactive type 2 , online interactive parameterized graph theory, algebra, artificial intelligence and operation-driven 4, various parameterization methods are shown in Table 1 below.

We can see that the above design methods are used more and more widely, especially in the field of industrial and architectural design, concept design, dynamic design, solid modeling, assembly tolerance analysis and synthesis, mechanism simulation, optimization design direction plays an increasingly important role, reflecting the very high application value.

\section{Parametric Modeling of Heavy Machine Parts}

About CATIA V5 kind of parametric design method in the above, it is found that the parameterization design method of third kinds of methods using spreadsheet driven graphics easily, this method is easy to grasp, easy to operate and design personnel, but also can easily achieve the desired series of standard parts. So the parametric model of the example uses the parametric method of "using the electronic form to drive the graph" to create the. Before the parametric design of parts, we must first consult data manual, structure size parts clear, and clearly understand the structure and use of painted parts, then the new parts file; then according to the main parameters of the structure and control of parts shape parts, determined the free parameters parts of the same parameters and the corresponding function, but also determine the relationship between the parameters; finally, to complete the detailed design of parts according to the preceding analysis. Below with heavy machine parts in the nut GB/T3632-2008 as an example, a brief introduction of parametric modeling.

Nut Custom Parameters. The basic parameters of bolt are: thread diameter (D), nut length (m), six head of edge width (S), nut mounting surface diameter (DW), bearing plane washer thickness (C), six head diagonal width (E), in the table to the $\mathrm{E}$ is the minimum, in order to convenient constraints, the direct use of the data in the table. Of course, it can also be based on the relationship between $\mathrm{E}$ and $\mathrm{S}$ to calculate the size of $\mathrm{E}$, such as Eq.1.

$$
e=2 \sqrt{\frac{S^{2}}{3}}
$$

According to the structural characteristics of the parts, the use of CATIA knowledge function comes with the parametric modeling function to complete the establishment of parametric model. From the initial size of the parts of the establishment of standards associated with the CATIA Excel form, it should be changed to correct specific header format, shown in Fig. 1.

\begin{tabular}{|c|c|c|c|c|c|c|c|}
\hline PartNumber & $\mathrm{D}(\mathrm{mm})$ & $\mathrm{P}(\mathrm{mm})$ & $\mathrm{e}(\mathrm{mm})$ & $\mathrm{m}(\mathrm{mm})$ & $\mathrm{C}(\mathrm{mm})$ & $\mathrm{S}(\mathrm{mm})$ & $\mathrm{dw}(\mathrm{mm})$ \\
\hline gbt3632-2008-Im_1 & 16 & 2 & 29.56 & 17.1 & 0.8 & 27 & 24.9 \\
\hline gbt3632-2008-Im_2 & 20 & 2.5 & 37.29 & 20.7 & 0.8 & 34 & 31.4 \\
\hline gbt3632-2008-Im_3 & 22 & 2.5 & 39.55 & 23.6 & 0.8 & 36 & 33.3 \\
\hline gbt3632-2008-Im_4 & 24 & 3 & 45.20 & 24.2 & 0.8 & 41 & 38 \\
\hline gbt3632-2008-Im_5 & 27 & 3 & 50.85 & 27.6 & 0.8 & 46 & 42.8 \\
\hline gbt3632-2008-Im_6 & 30 & 3.5 & 55.37 & 30.7 & 0.8 & 50 & 46.5 \\
\hline
\end{tabular}

Figure 1. Nut parameter library

In the CATIA tool formula, the geometric parameters, dimensionless parameters and physical parameters of the parts are created, and the geometric parameters are defined as the "length". The parameters of the definition are shown in Fig. 2. 


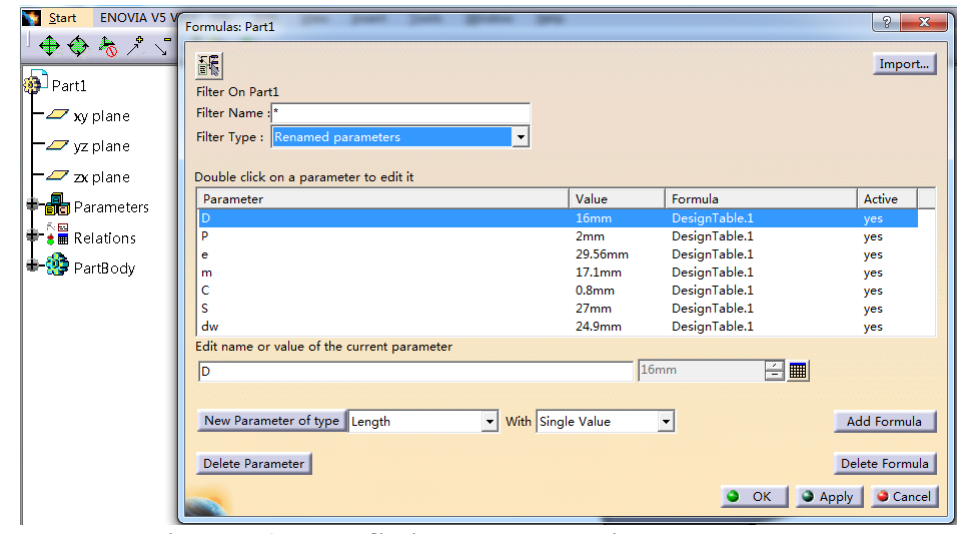

Figure 2. Defining geometric parameters

\section{Sketch and Constrain}

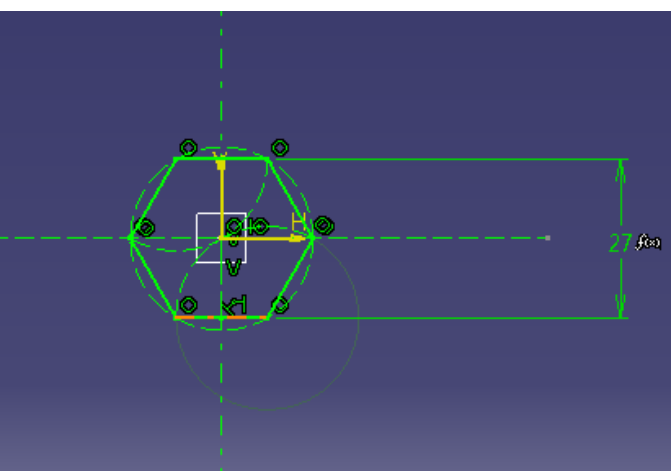

Figure 3. Nut fully constrains the sketch 1

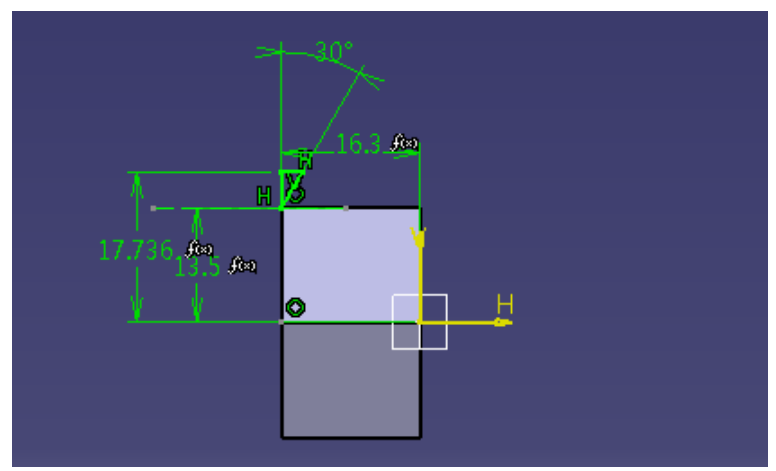

Figure 4. Nut fully constrains the sketch 2

First of all, the shape of the cross-section of the nut is drawn. The specific shape and size of the part outline are all guaranteed by adding constraints. He constraints in the contour are: (1)the opposite sides of the hexagon are parallel to each other; (2)each side length is equal, Finally, the size of the hexagon with the parameter $\mathrm{S}$ constraints, thus completing the complete constraints on the sketch. All the lines will be shown in green, as shown in Fig. 3; and then in order to draw the shape of the nut surface, draw a small triangle in the sketch, fully constrained to get Fig. 4, and then rotate the cut, the groove of the command, the final results are shown in Fig. 5.

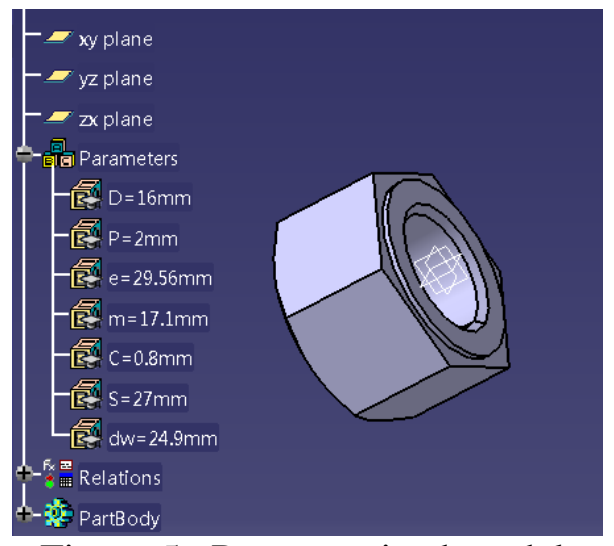

Figure 5. Parameterized model

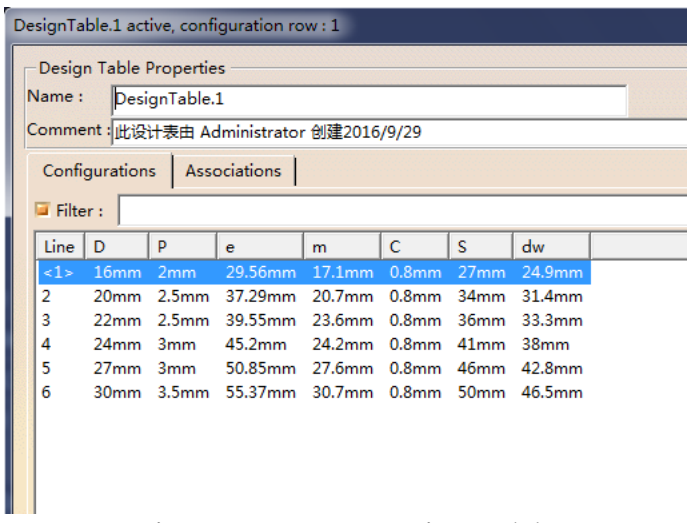

Figure 6. Nut series table 
Association Table and Analysis of Part Family. In CATIA V5, there are three ways to build standard parts library: (1) Using the Formula, Design Table and Catalog functions provided by CATIA software, to build and use 3D standard parts library;(2)To use VB to provide reference library, add CATIA library file The program framework, the reference CATIA class objects and functions, such as secondary development;3)CATIA provided by the application of the secondary development tools RADE and library functions CAA interface, in the VC development environment to create standard parts library applications.

In this paper, the first method is used to build the standard parts library, using CATIA knowledge function "Design Table Design Table" function to add the previously compiled driver parameter table, shown in Fig. 1, after a good correlation into the series table shown in Fig. 6. The nut of the parametric model into the Catalog template, the final analysis to generate a family of parts as shown in Fig. 7.

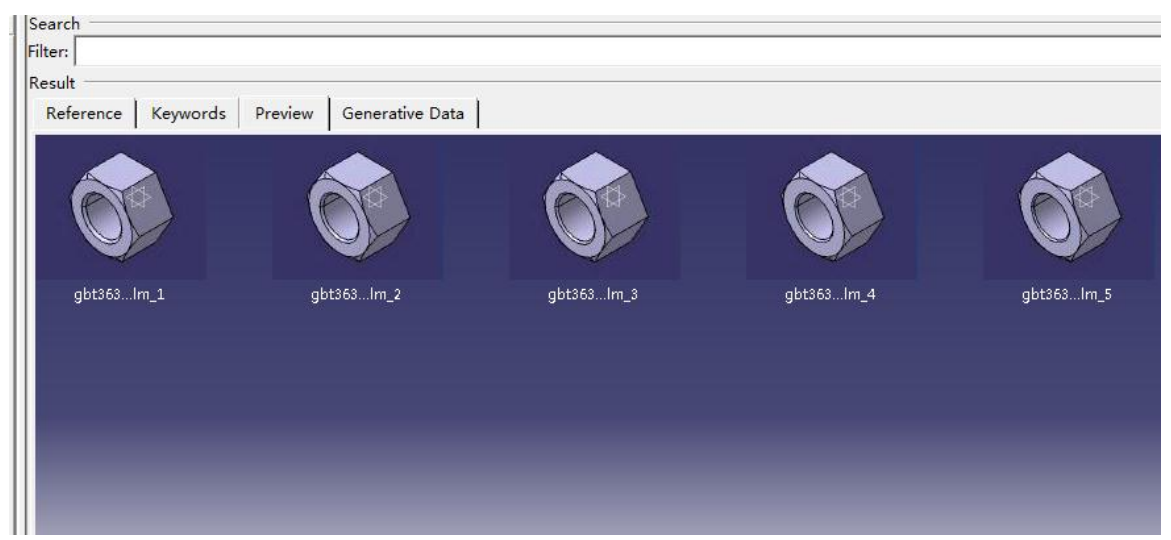

Figure 7.

For the use of standard parts in the standard parts library, you can directly open the corresponding icon in the standard parts library. Line parameters can be modified to obtain the corresponding standard parts.

\section{Summary and Prospect}

Although the parametric technology from the initial sprout gradually developed and achieved considerable results, but from the practical application of the situation, there are several parameters of the following technical problems: (1)the parametric graphics processing is relatively simple to handle such as section lines, process roughness and other complex annotation and the direction constraint; (2)with the solution of the lack of effective solutions to the constraint graph with cyclic constraints; (3)the parts inside the product and how to cross between different product realization and the relationship between the parameters of the drive.

In this paper, the nut as an example, introduced in the CATIA V5 environment, this paper introduces the design method, parametric design ideas and design process based on series parts were designed by CATIA software, to achieve fast and accurate design of parts drawing. It can be seen that the parametric design can enable designers to realize parametric database in the design at the same time, which greatly facilitates the subsequent design work, to further it is of great significance to improve drawing efficiency and design and flexible design. Although there are some problems in the process of establishing parametric software system. Along with the mutual integration of various parameters, the continuous development of various new technologies, we believe that the final will be able to overcome the above problems, improve the parameterization of technology. 


\section{Acknowledgements}

The paper is supported by Key Problem Tackling Project of Shaanxi Scientific and Technological Office (2016GY-024).

\section{References}

[1] J. Li, G.D. Zhao: Parametric modeling and design of 3D parts [J]. Premiere, 2015, 12:28.

[2] J.W. Li: Parameterized Design Study on Mechanical Devices [A]. Electronic mechanical engineering branch of China Electronic Society. Proceedings of the Conference on Mechanics and Electronics[C] 2011.2011:5.

[3] Y.H. Shen, L.H. Zou, L.Q. Dai, X.Y. Zhao, F.Q. Zhao: Parameterized Design and Database Set up of Standardized Parts Based on CATIA [J].Auto Engineer, 2012, 09:53-55.

[4] G.Y. Wang: Parametric design analysis and research of automobile gear rack type steering gear [D].Shenyang University of Technology.2013

[5] H.J. Dong, X.M. Jing, Y.J Guo, T. Zang, Z. Fang:Parametric Design for Contact of Vacuum Circuit Breaker Based on Solid Works, 2014,10:1135-1139.

[6] L.M. Wang: Aircraft parametric 3D shape design and 3D reconstruction based on CAA CATIA [D]. Nanjing University of Aeronautics and Astronautics, 2014

[7] Q.S. Wu: Parametric design of aircraft shape feature based on knowledge [D]. Nanchang Hangkong University

[8] Y.G Wang, L.J Yang: Three-dimensional Parametric Modeling Technology and Application Based on CATIA [J]. Mechanical Engineer, 2014, 05:200-202.

[9] Z.G Liang, G.D. Sun: 3D parametric modeling technology for aircraft parts based on CATIA/CAA [J]. Journal of Sichuan Ordnance, 2012, 03:72-74

[10]X.Y. Wang: 3D Parametric Modeling Method and Application Based on CATIA [J]. Auto Parts, 2012, 03:55-58. 